\title{
Características agronómicas asociadas a la producción de bioetanol en genotipos de sorgo dulce ${ }^{1}$
}

\section{Agronomic characteristics associated with bioethanol production in sweet sorghum genotypes}

\author{
Héctor Williams-Alanís², Francisco Zavala-García², Gerardo Arcos-Cavazos ${ }^{3}$, \\ María del Carmen Rodríguez-Vázquez², Emilio Olivares-Sáenz²
}

\begin{abstract}
Resumen
La producción de energía a partir de bioetanol de sorgo, es una manera de ayudar a aminorar el cambio climático y el deterioro del ambiente. El objetivo del trabajo fue evaluar las características agronómicas asociadas a la producción de bioetanol en genotipos experimentales de sorgo dulce. Durante los años 2013 y 2014 se evaluaron diecisiete genotipos dulces en dos localidades del noreste de México, bajo un diseño de bloques completos al azar con tres repeticiones: tres experimentos en la Estación Cuauhtémoc, Tamaulipas, en temporal; y dos en Marín, Nuevo León, en riego. El híbrido experimental Potranca x Keller obtuvo los valores más altos $(\mathrm{p} \leq 0,05)(\mathrm{kg} / \mathrm{ha})$ en: peso de biomasa (52 125), de tallo (39 650), de espiga (4550), de hoja (7700), de jugo (14 125) y de bioetanol (2157 l/ha); y superó al testigo más productivo (Keller) en: 35,7, 32,5, 63,2, 41,6, 36,8 y 32,5\%, respectivamente. Además, fue 6,8 días más precoz en cuanto a la floración que el testigo. La variedad experimental 17-1-1-1 se identificó con menor incidencia a enfermedades foliares que Keller. Además, presentaron características agronómicas similares al testigo en: peso de biomasa (44 $375 \mathrm{~kg} / \mathrm{ha})$, de tallo (35 $438 \mathrm{~kg} / \mathrm{ha})$, de espiga (2488 kg/ha), de hoja (6400 kg/ha), de jugo $(11750 \mathrm{~kg} / \mathrm{ha})$, peso seco de planta (19 $113 \mathrm{~kg} / \mathrm{ha})$, peso seco de tallo (14 $888 \mathrm{~kg} / \mathrm{ha})$, producción de bioetanol (1929 $1 /$ ha), ${ }^{\circ}$ Brix $(15,2)$, días a floración $(80,8)$ y altura de planta $(224 \mathrm{~cm})$.
\end{abstract}

Palabras clave: Sorghum bicolor, mejoramiento genético, energía renovable, producción de biomasa.

\begin{abstract}
Energy production from sorghum bioethanol is a way to help decrease climate change and environmental degradation. The objective of this work was to evaluate the agronomic characteristics associated to bioethanol production in experimental genotypes of sweet sorghum. During 2013 and 2014, seventeen sweet genotypes were evaluated in two locations, this locations are situated in northeastern Mexico, under a complete block randomly design with three replications. The three experiments were executed in Estacion Cuauhtemoc, Tamaulipas, in
\end{abstract}

1 Recibido: 27 de octubre, 2016. Aceptado: 2 de marzo, 2017. Proyecto de investigación No. 227422 "Sweetfuel sorghum: an alternative energy crop", financiado por la Comunidad Económica Europea.

2 Universidad Autónoma de Nuevo León (UANL), Facultad de Agronomía. km 17.5 Carretera Zuazua-Marín, Marín, N. L., México. 66700. hectorwilliamsa@yahoo.com.mx (autor para correspondencia),francisco.zavala.garcia@gmail.com, mary-carmen72@hotmail.com, emolivares@gmail.com

3 Instituto Nacional de Investigaciones Forestales, Agrícolas y Pecuarias (INIFAP), Campo Experimental Huastecas. Carretera Tampico-Cd. Mante Km. 55 Apdo. postal C-1 Suc. Aeropuerto, Tampico, Tam., México. CP 89339. arcos.gerardo@inifap.gob.mx 
dryland; and two in Marin, Nuevo León, under irrigation. Experimental hybrid Potranca x Keller ( $\mathrm{p} \leq 0.05)$ presented the highest values (kg/ha) in: biomass weight (52 125), stem (39 650), panicle (4550), leaf (7700), juice (14 125), and bioethanol (2157 1/ha); it surpassed to the most productive control (Keller) in: 35,7, 32,5, 63,2, 41,6, 32,5, and $36,8 \%$, respectively. It bloomed 6.8 days earlier than variety control. Experimental variety 17-1-1-1 had a minor foliar incidence of diseases in comparison to Keller. In addition, it presented agronomic characteristics similar to control in: biomass weight (44 $375 \mathrm{~kg} / \mathrm{ha})$, stem (35 $438 \mathrm{~kg} / \mathrm{ha})$, panicle (2488 kg/ha), leaf (6400 kg/ha), juice (11 $750 \mathrm{~kg} / \mathrm{ha})$, plant dry weight (19 $113 \mathrm{~kg} / \mathrm{ha})$, stem dry weight (14 $888 \mathrm{~kg} / \mathrm{ha})$, bioethanol production $(1929 \mathrm{l} / \mathrm{ha})$, ${ }^{\circ}$ Brix $(15,2)$, days to flowering $(80,8)$ and plant height $(224 \mathrm{~cm})$.

Keywords: Sorghum bicolor, sorghum breeding, renewable energy, biomass production.

\section{Introducción}

Debido al inminente agotamiento de las reservas de combustibles fósiles como el petróleo, el gas natural y el carbón, y las consecuencias de su uso indiscriminado en el deterioro ambiental, se espera que los biocombustibles contribuyan cada vez más en suplir las necesidades de energía del mundo (Sun et al., 2014). Estos son una alternativa para la reducción de los gases de efecto invernadero (GEI) y la mitigación del cambio climático, y amigables con el ambiente, debido a que las emisiones de $\mathrm{CO}_{2}$ que se producen durante la combustión, son compensadas por el que es absorbido durante el crecimiento de las plantas (Almodares y Hadi, 2009; Khawaja, et al., 2014).

El sorgo (Sorghum bicolor L. Moench) es uno de los cultivos más antiguos y actualmente uno de los cereales de mayor importancia en el mundo (Serna, 2010). Puede producirse en todos los continentes, en regiones tropicales, subtropicales y templadas; en tierras de mediana y baja aptitud agrícola, y especialmente apto para regiones con escasa precipitación (Tobin, 2010). En México el grano de sorgo, es el principal ingrediente en la formulación de alimentos balanceados para animales. La superficie dedicada a este cultivo alcanzó un promedio de dos millones de hectáreas en los últimos diez años (SIAP, 2017).

S. bicolor pertenece a las plantas $\mathrm{C} 4$, las cuales forman compuestos de cuatro carbonos, haciéndolas más eficientes en el uso del agua, dióxido de carbono y nutrientes (Khawaja et al., 2014). El sorgo dulce pertenece a la misma especie que el sorgo de grano, forrajero y escobero, pero ha sido seleccionado por acumular altos niveles de sacarosa en los tallos (Vietor y Miller, 1990; Murray et al., 2009). Es considerado la mejor opción para producir bioetanol en lugar de maíz (Zea mays L.) y caña de azúcar (Saccharum officinarum L.), debido a que presenta numerosas ventajas: es tolerante a sequía, salinidad, altas temperaturas (Murray, 2008; Chuck et al., 2011) y suelos inundados (Almodares et al., 2007); es de ciclo de crecimiento corto y presenta amplia adaptación. Además, su siembra a partir de semilla es fácil y barata, y la cosecha es mecanizada (Murray, 2008); es uno de los cultivos de mayor eficiencia en el uso de la radiación solar (1,4 -2,8 g biomasa/MJ); su bagazo y los residuos de cosecha tienen un valor bioenergético similar al de la caña de azúcar (Tobin, 2010). De acuerdo con Serna (2010), estas podrían ser las "plantas del futuro", debido a la escasez de agua disponible para irrigación y el mayor nivel de $\mathrm{CO}_{2}$ presente en el ambiente.

El sorgo dulce (S. bicolor), a diferencia del maíz y la caña de azúcar, cuenta con poco historial en el mejoramiento genético, lo que significa que tiene un gran potencial sin explotar (Ratnavathi et al., 2010). Presenta una amplia gama de variabilidad genética en características morfológicas tales como altura de planta, grosor del tallo y ciclo vegetativo, que tienen una relación directa con la producción de bioetanol (Reddy et al., 2005), lo cual hace imperativo generar nuevos cultivares de sorgo dulce, con alto contenido de azúcar en combinación con otros caracteres agronómicos deseables (Ali et al., 2008). El primer cultivar de S. bicolor introducido en USA fue la variedad china Amber en 1853, que se utilizó para producir jarabe (Khawaja et al., 2014); desde entonces, muchas 
otras variedades dulces de S. bicolor fueron introducidas de otros países o desarrolladas localmente (Undersander et al., 1990).

A partir del año 1944, la Oficina de Estudios Especiales realizó las primeras investigaciones en S. bicolor en México (Reyes, 1985), se publicaron las primeras recomendaciones y prácticas de cultivo en 1950 (Williams et al., 2006). Posterior a esta fecha, se realizaron numerosas introducciones de genotipos de sorgo de grano, forrajero, escobero y dulces. En los inicios de los años 2000, empezó a cobrar interés el S. bicolor para producción de bioetanol, por lo cual, se empezaron a introducir nuevos genotipos de USA, Asia y África, iniciando con ellos algunos trabajos de investigación en agronomía y mejoramiento genético. En el año 2010, el Campo Experimental Rio Bravo (INIFAP) generó la variedad dulce RB-Cañero y las recomendaciones técnicas para su producción comercial (Montes et al., 2010).

El etanol es el biocombustible más utilizado en el mundo y es usado ampliamente en Brasil y Estados Unidos (Yuvraj et al., 2013). También es conocido como alcohol etílico o alcohol de grano y producido por la fermentación de azúcares derivados del trigo (Triticum aestivum), maíz, remolacha [Beta vulgaris (L.)] y caña de azúcar (Yuvraj et al., 2013). La ventaja del etanol con respecto a los combustibles fósiles, es que se obtiene de fuentes renovables (Almodares y Hadi, 2009; Chuck et al., 2011). Puede ser utilizado como combustible en forma pura (Yuvraj et al., 2013), pero es más común utilizarlo como sustituto del metil tert-butil éter (MTBE), oxigenante de la gasolina altamente tóxico, tan soluble en el agua, que representa en la actualidad, una de las fuentes más peligrosas de contaminación de los mantos acuíferos (Almodares y Hadi, 2009; Serna, 2010; Yuvraj et al., 2013).

En México, a raíz de la reforma energética promulgada en el año 2013, se abrió la posibilidad de producir biocombustibles a partir de caña de azúcar, sorgo dulce, agave (Agave americana L.), remolacha, jatropha (Jatropha curcas L.) e higuerilla (Riccinus communis L.) (Zafranet, 2015). Se estima que Petróleos Mexicanos (PEMEX), podrán adquirir hasta 123 millones de litros de etanol anhidro al año, de productores mexicanos de sorgo y caña de azúcar (PEMEX, 2015). De tal forma que, para el año 2017, se prevé que doce plantas estén produciendo biocombustibles y contemplando áreas de producción en Veracruz, Jalisco, Chiapas, Tabasco, Tamaulipas y Colima (Zafranet, 2015). Considerando los planes y políticas oficiales, los investigadores se deben de enfocar en: la innovación de mejores prácticas agronómicas para su cultivo y producción, reducir costos, aumentar la productividad y generar genotipos más eficientes en el aprovechamiento del espacio, agua y nutrientes. Atendiendo a la problemática descrita, se diseñó el presente trabajo de investigación, con el objetivo de evaluar las características agronómicas asociadas a la producción de bioetanol en genotipos experimentales de sorgo dulce.

\section{Materiales y métodos}

\section{Generación de variedades experimentales}

Con el objeto de aumentar la variabilidad genética e iniciar el proceso de selección de nuevos genotipos, durante el ciclo primavera 2010 se realizó la cruza entre las líneas SBB-25 × Rox Orange. SBB-25 es una línea de grano élite mantenedora de la fertilidad, desarrollada en el INIFAP, Campo Experimental Río Bravo, progenitora de los híbridos comerciales RB-4000 y RB-Patrón (Williams et al., 2004), y RB-Huasteco (Montes et al., 2014). Rox Orange o Waconia, variedad comercial de sorgo forrajero y dulce, generada por Wisconsin Agricultural Experiment Station en Madison, USA (Undersander et al., 1990). En la generación F2 de la cruza, cuando las plantas estaban entre las etapas de grano lechoso y masoso, fueron identificadas 32 plantas con un contenido de azúcar en el jugo entre 15 a $20,5^{\circ}$ Brix. Se cosechó el grano de las plantas individuales para continuar con el proceso de selección. A partir de estas semillas en las generaciones F3, F4 y F5, se realizó una selección de surco por panoja o pedigrí, autofecundando plantas altas y jugosas, con alto contenido de azúcar ( $+15^{\circ}$ Brix), tallo más 
grueso, sin acame y barrenador, y tolerantes a enfermedades foliares, obteniendo a finales del año 2012 un grupo de variedades experimentales.

\section{Formación de Potranca}

Durante los años 2008 a 2012, en un proceso de retrocruzamiento, utilizando el método de selección apareada de progenie descrito por House (1985), se obtuvo a Potranca (línea androestéril). Durante el proceso de obtención, se utilizó como progenitor donante a 46038, línea de grano con androesterilidad genética citoplásmica, desarrollada por la Universidad de Lincoln, Nebraska, USA. La androesterilidad introducida del tipo A1, pertenece a la descrita por primera vez por Stephens y Holland (1954). Como progenitor recurrente se usó a Rox Orange, variedad de sorgo dulce y forrajera (S. bicolor), que se comporta como mantenedora de la fertilidad. En el ciclo primavera 2012, al término de siete retrocruzas hacia su progenitor recurrente, se obtuvo a Potranca, la cual se registró en noviembre del 2014 con el número CP-1081, en el Catálogo Nacional de Variedades Vegetales (CNVV).

\section{Ambientes de evaluación}

La investigación se realizó en dos localidades (cinco experimentos) del noreste de México: Campus Marín, primavera 2014 (MP-2014) y verano 2014 (MV-2014); y Estación Cuauhtémoc, verano 2013 (EC-V2013), otoñoinvierno 2014 (ECOI-2014) y verano 2014 (ECV-2014).

El Campus Marín, de la Facultad de Agronomía de la Universidad Autónoma de Nuevo León (FAUANL), se localiza en el km 17 de la carretera Zuazua-Marín, en el municipio de Marín, Nuevo León. Geográficamente se ubica en las coordenadas $25^{\circ} 53^{\prime} \mathrm{LN} / 100^{\circ} 02^{\prime} \mathrm{LO}$, a una altura de $355 \mathrm{msnm}$. El clima corresponde a un BS1 (h) $\mathrm{w}(\mathrm{e})$, descrito como clima seco estepario cálido con lluvias en verano, con una precipitación promedio anual de 595 mm y temperatura media anual de $22{ }^{\circ} \mathrm{C}$ (García, 1988). El tipo de suelo es vertisoles, delgados, con alto contenido de arcilla y carbonato de calcio, con bajos contenidos de materia orgánica y pH entre 7,5 a 8,5.

El sitio experimental localizado en la Estación Cuauhtémoc, municipio de Altamira, Tamaulipas, en terrenos del Campo Experimental las Huastecas, INIFAP. Se ubica en las coordenadas $22^{\circ} 33^{\prime} \mathrm{LN} / 98^{\circ} 09^{\prime} \mathrm{LO}$, con una altura de $20 \mathrm{msnm}$. Presenta un clima $\left(\mathrm{Aw}_{0}\right)$ cálido subhúmedo con lluvias en verano y lluvia invernal de 5-10\% (García, 1988), con promedio de temperatura media anual de $24,5^{\circ} \mathrm{C}$ y $842 \mathrm{~mm}$ de precipitación (INIFAP, 2013). El tipo de suelo es vertisol pélico arcilloso y profundo, con un $\mathrm{pH}$ de 7,5 a 7,8.

\section{Manejo de los experimentos}

Los experimentos se establecieron en ensayos uniformes con diecisiete genotipos, en un diseño de bloques completos al azar y tres repeticiones. Los genotipos fueron: diez variedades experimentales originadas de la cruza de SBB-25 x Rox Orange, Potranca (línea androesteril), dos híbridos experimentales producto de la cruza de Potranca con Keller y Urja (Cuadro 1), y cuatro variedades comerciales: Rox Orange y Keller (USA); Urja (India) y RB-Cañero (México). Incluyendo dentro de estas a los testigos (T); Keller, Urja y RB-Cañero.

La parcela experimental consistió de un surco de $5 \mathrm{~m}$ de largo y $0,80 \mathrm{~m}$ de separación, con un área de $4 \mathrm{~m}^{2}$. En Marín los experimentos se condujeron en condiciones de riego. Antes de la siembra se aplicó al suelo y al voleo, 2 t de gallinaza/ha, la cual se realizó en seco, mediante una sembradora de experimentos.

En Estación Cuauhtémoc, los ensayos se condujeron en condiciones de temporal y la siembra se realizó en tierra húmeda, aplicando la semilla a chorrillo a mano en el fondo del surco. Se realizó una fertilización dividida en dos partes con la fórmula 90-40-00, elaborada con superfosfato triple y nitrato de amonio. La primera antes de 
Cuadro 1. Relación de genotipos de sorgo dulce evaluados en dos localidades del noreste de México, durante los años 2013 y 2014. Table 1. Sweet sorghum genotypes evaluated in two locations in northeastern Mexico during 2013 and 2014.

\begin{tabular}{|c|c|c|c|}
\hline Número & Genealogía & Número & Genealogía \\
\hline 1 & $3-4-1-1^{a}$ & 10 & $22-2-2-1^{a}$ \\
\hline 2 & $4-1-1-1^{a}$ & 11 & Potranca $^{\mathrm{b}}$ \\
\hline 3 & $4-2-1-1^{a}$ & 12 & Potranca x Keller ${ }^{c}$ \\
\hline 4 & $17-1-1-1^{a}$ & 13 & Potranca $x U{ }^{\prime} j a^{c}$ \\
\hline 5 & $31-2-1-2^{a}$ & 14 & Rox Orange $^{\mathrm{d}}$ \\
\hline 6 & $4-5-1-1^{\mathrm{a}}$ & 15 & $\operatorname{Keller}^{\mathrm{d}}(\mathrm{T})$ \\
\hline 7 & $5-5-1-1-1 \mathrm{PL}^{\mathrm{a}}$ & 16 & $\operatorname{Urja}^{\mathrm{d}}(\mathrm{T})$ \\
\hline 8 & $17-1-2-1^{\mathrm{a}}$ & 17 & RB-Cañero ${ }^{d}(T)$ \\
\hline 9 & $20-3-1-1-2^{\mathrm{a}}$ & & \\
\hline
\end{tabular}

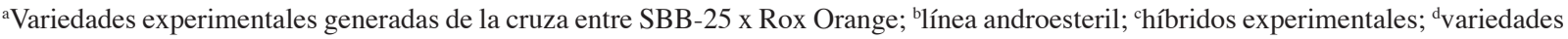

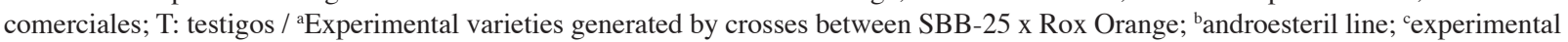
hybrids; ${ }^{\mathrm{d}}$ comercial varieties; T: control.

la siembra donde se aplicó la mitad del nitrógeno y todo el fósforo, y la segunda aplicación a los quince días de la siembra con la otra mitad del nitrógeno. A los veinte días de nacidas las plantas, se dejó en ambas localidades una población de 150000 plantas/ha.

\section{Toma de datos}

Se cosecharon las plantas al final de la etapa siete (grano lechoso), descrita por Vanderlip (1993), periodo en el cual alcanzan el máximo de azúcar en el jugo del tallo (Dávila et al., 2011), en un área de $0,8 \mathrm{~m}^{2}$ de la parcela, cortando las plantas de 3 a $4 \mathrm{~cm}$ de la base del tallo, donde se tomaron los siguientes datos (g): peso total de planta (PTP), peso del tallo (PT), peso de hoja (PH), peso de espiga (PE), peso seco total de planta (PSP) y peso seco de tallo (PST). Estos últimos dos valores se obtuvieron al secar y pesar el peso fresco de tallo, espiga y hojas en una estufa a $65^{\circ} \mathrm{C}$ por tres días. En Marín se extrajo el jugo de los tallos, con un extractor eléctrico, donde se sacó el peso de jugo en $\mathrm{g}(\mathrm{PJ}), \mathrm{y}$ con un refractómetro manual los ${ }^{\circ} \mathrm{Brix}$. El valor de días a floración (DF), se tomó cuando el $50 \%$ de las plantas estaban en floración y una semana antes de la madurez fisiológica, se midió la altura de planta (AP) en cm, desde el suelo hasta el ápice de la panoja.

En la Estación Cuauhtémoc se obtuvo los ${ }^{\circ}$ Brix del jugo, midiendo y promediando cinco plantas por parcela en el entrenudo medio de la planta, con el mismo tipo de refractómetro. En las localidades de la Estación Cuauhtémoc, el peso de jugo (PJ) se estimó con la siguiente fórmula: PJ = PT x \% J/100. El \% de jugo (\% J) por genotipo se obtuvo en Marín multiplicando el PJ x 100 y dividiéndolo entre PT. Para todas la características evaluadas, los valores obtenidos de peso en gramos se transformaron a $\mathrm{kg} / \mathrm{ha}$. La producción de bioetanol (1/ha), se estimó multiplicando el peso fresco de tallo en $\mathrm{kg} / \mathrm{ha}$ por 0,0544 , de acuerdo con lo reportado por Almodares y Hadi (2009). La incidencia de enfermedades foliares (IEF) se evaluó en las localidades de Estación Cuauhtémoc, y el acame (A) en Marín verano 2014. La primera característica se calificó en forma visual, mediante una escala de 1 al 5, donde: 1 es sin daño, 2 de 1 a 10\% de daño, 3 de 11 a 25\% de daño; 4 de 26 a $40 \%$ de daño y 5 más de $41 \%$. Para acame: 1 es 10\% de plantas acamadas, 2 es de 10 a 25\% de plantas acamadas, 3 de 25-50\%, 4 de 50-75\% y 5 de $75-100 \%$. Esto en concordancia con las indicaciones descritas por House (1985). Se utilizó la información 
meteorológica (precipitacion, temperatura, etc.), que se obtuvo de las estaciones ubicadas en Marín, Nuevo león y Estación Cuauhtémoc, Tamaulipas.

\section{Análisis estadístico}

El efecto del ambiente en el rendimiento y las características agronómicas, se cuantificaron mediante un análisis combinado en los cinco experimentos y los diecisiete genotipos, utilizando el programa SPSS, y la comparación de medias se realizó con la prueba de Tukey al 0,05 de probabilidad.

\section{Resultados}

\section{Análisis combinado}

En el análisis de varianza combinado de los cinco experimentos, todas las variables evaluadas mostraron diferencias altamente significativas para ambientes, genotipos y la interacción ambientes x genotipos (Cuadro 2).

Los valores más altos para PJ, PTP, PT, DF, PE, PH, PSP PST y BI se presentaron para las localidades de ECOI-2014 y ECV-2014 (Cuadro 2). Para ${ }^{\circ}$ Brix se presentaron diferencias, observándose los valores mayores $(16,2)$ para MP-2014 y mínimos para MV-2014 $(13,1)$, ECOI-2014 $(14,3)$ y ECV-2013 $(14,6)$.

Cuadro 2. Comparación de medias (Tukey) de ambientes para variables de diecisiete genotipos de sorgos dulces evaluados en dos localidades del noreste de México, durante los años 2013 y 2014.

Table 2. Comparison variables regarding environmental means, where seventeen sweet sorghum genotypes were evaluated at northeastern Mexico, during 2013 and 2014.

\begin{tabular}{lccccc}
\hline & \multicolumn{5}{c}{ Localidades } \\
\cline { 2 - 6 } Características & ECOI-2014 & ECV-2014 & MV-2014 & MP-2014 & ECV-2013 \\
\hline PJ & $12863 \mathrm{a}$ & $11875 \mathrm{a}$ & $9463 \mathrm{~b}$ & $8075 \mathrm{~b}$ & $7625 \mathrm{c}$ \\
${ }^{\circ}$ Brix & $14,3 \mathrm{c}$ & $15,4 \mathrm{ab}$ & $13,1 \mathrm{~d}$ & $16,2 \mathrm{a}$ & $14,6 \mathrm{bc}$ \\
PTP & $49000 \mathrm{a}$ & $52425 \mathrm{a}$ & $37812 \mathrm{~b}$ & $31525 \mathrm{bc}$ & $30663 \mathrm{c}$ \\
PT & $37238 \mathrm{a}$ & $40163 \mathrm{a}$ & $30425 \mathrm{~b}$ & $24025 \mathrm{c}$ & $23950 \mathrm{c}$ \\
DF & $83,4 \mathrm{a}$ & $75,6 \mathrm{c}$ & $69,7 \mathrm{~d}$ & $82,4 \mathrm{~b}$ & $84,8 \mathrm{a}$ \\
AP & $231 \mathrm{~b}$ & $*$ & $259 \mathrm{a}$ & $197 \mathrm{~d}$ & $209 \mathrm{c}$ \\
PE & $375 \mathrm{a}$ & $3875 \mathrm{a}$ & $1338 \mathrm{~d}$ & $1888 \mathrm{bc}$ & $2313 \mathrm{~b}$ \\
PH & $6913-\mathrm{b}$ & $8388 \mathrm{a}$ & $5175 \mathrm{de}$ & $5288 \mathrm{~cd}$ & $4750 \mathrm{e}$ \\
PSP & $21200 \mathrm{~b}$ & $24813 \mathrm{a}$ & $7763 \mathrm{~d}$ & $*$ & $* 38 \mathrm{c}$ \\
PST & $15500 \mathrm{~b}$ & $19725 \mathrm{a}$ & $5363 \mathrm{c}$ & $*$ & $13900 \mathrm{~b}$ \\
BI & $2025 \mathrm{a}$ & $2185 \mathrm{a}$ & $1655 \mathrm{~b}$ & $1307 \mathrm{c}$ & $1303 \mathrm{c}$ \\
IEF & $2,8 \mathrm{a}$ & $1,9 \mathrm{c}$ & $*$ & $*$ & $2,4 \mathrm{~b}$ \\
\hline
\end{tabular}

*No se obtuvo información. ECOI-2014: Estación Cuauhtémoc otoño-invierno 2014. Fecha de siembra 23 de diciembre; ECV-2014: Estación Cuauhtémoc verano 2014. Fecha de siembra 9 de septiembre; MV-2014: Marín verano 2014. Fecha de siembra 23 de agosto; MP-2014: Marín primavera 2014. Fecha de siembra 20 de marzo; ECV-2013: Estación Cuauhtémoc verano 2013. Fecha de siembra 19 de agosto / *No information was obtained. ECOI-2014: Estacion Cuauhtemoc, fall-winter, 2014. Planting date: December 23; ECV2014: Estacion Cuauhtemoc, summer, 2014. Planting date: September 9; MV 2014: Marin, summer, 2014. Planting date: August 23; MP-2014: Marin, spring, 2014. Planting date: March 20; ECV-2013: Estacion Cuauhtemoc, summer, 2013. Planting date: August 19. 


\section{Peso total de planta (PTP), peso de tallo (PT) y peso de espiga (PE)}

Potranca x Keller, presentó valores superiores de las variables PTP, PT y PE con respecto a los testigos (Cuadro 3). Este híbrido fue mejor que Keller, Urja y RB-Cañero, superando al mejor testigo Keller en 35,8, 32,5 y $63,2 \%$, respectivamente en estas características. También se observó que las variedades experimentales: 5-5-11-1PL, 22-2-2-1 y 17-1-1-1 fueron iguales a Keller y Urja en PTP, PT y PE. Las variedades Rox Orange, 3-4-1-1 y RB-cañero fueron las que presentaron los valores más bajos en PTP y PT. El mayor peso de espiga lo presentó Potranca x Keller con 4550 (kg/ha) y el menor valor lo presentó Potranca con (1775 kg/ha).

Cuadro 3. Características de planta de diecisiete genotipos de sorgos dulces evaluados en dos localidades del noreste de México, durante los años 2013 y 2014.

Table 3. Plant characteristics of seventeen sweet sorghums genotypes, evaluated in two locations of northeastern Mexico, during 2013 and 2014.

\begin{tabular}{lcccccc}
\hline Genotipo & PTP & PT & PE & PH & PSP & PST \\
\hline Potranca x Keller & $52125 \mathrm{a}$ & $39650 \mathrm{a}$ & $4550 \mathrm{a}$ & $7700 \mathrm{a}$ & $20875 \mathrm{abc}$ & $15163 \mathrm{abcd}$ \\
$5-5-1-1-1 P L$ & $44475 \mathrm{abc}$ & $35825 \mathrm{abc}$ & $3625 \mathrm{abc}$ & $5875 \mathrm{ab}$ & $21750 \mathrm{ab}$ & $17088 \mathrm{ab}$ \\
$17-1-2-1$ & $47475 \mathrm{ab}$ & $37500 \mathrm{ab}$ & $2238 \mathrm{de}$ & $7400 \mathrm{a}$ & $23163 \mathrm{a}$ & $18650 \mathrm{a}$ \\
$22-2-2-1$ & $41475 \mathrm{abc}$ & $32550 \mathrm{abcd}$ & $2700 \mathrm{cde}$ & $6238 \mathrm{ab}$ & $17788 \mathrm{abcd}$ & $14013 \mathrm{abcde}$ \\
$17-1-1-1$ & $44375 \mathrm{abc}$ & $35438 \mathrm{abc}$ & $2488 \mathrm{cde}$ & $6400 \mathrm{ab}$ & $19113 \mathrm{abcd}$ & $14888 \mathrm{abcd}$ \\
Keller (T) & $38413 \mathrm{bc}$ & $29925 \mathrm{bcd}$ & $2788 \mathrm{cde}$ & $5438 \mathrm{~b}$ & $18400 \mathrm{abcd}$ & $14375 \mathrm{abcd}$ \\
$4-2-1-1$ & $41238 \mathrm{abc}$ & $31975 \mathrm{abcd}$ & $1988 \mathrm{de}$ & $6775 \mathrm{ab}$ & $18613 \mathrm{abcd}$ & $15200 \mathrm{abcd}$ \\
Potranca x Urja & $41538 \mathrm{abc}$ & $31413 \mathrm{abcd}$ & $3613 \mathrm{abc}$ & $6300 \mathrm{ab}$ & $17513 \mathrm{abcd}$ & $15938 \mathrm{abc}$ \\
Urja (T) & $42288 \mathrm{abc}$ & $33988 \mathrm{abcd}$ & $2488 \mathrm{cde}$ & $5950 \mathrm{ab}$ & $19988 \mathrm{abcd}$ & $15938 \mathrm{abc}$ \\
Potranca & $36000 \mathrm{bc}$ & $28013 \mathrm{bcd}$ & $1775 \mathrm{e}$ & $5788 \mathrm{ab}$ & $15775 \mathrm{bcd}$ & $12663 \mathrm{bcde}$ \\
$20-3-1-1-2$ & $39088 \mathrm{bc}$ & $29113 \mathrm{bcd}$ & $3175 \mathrm{bcd}$ & $6700 \mathrm{ab}$ & $13963 \mathrm{~cd}$ & $10300 \mathrm{de}$ \\
Rox Orange & $35200 \mathrm{~d}$ & $26750 \mathrm{~cd}$ & $2063 \mathrm{de}$ & $5875 \mathrm{ab}$ & $14725 \mathrm{~cd}$ & $11450 \mathrm{cde}$ \\
$4-1-1-1$ & $37288 \mathrm{bc}$ & $28788 \mathrm{bcd}$ & $1950 \mathrm{de}$ & $6312 \mathrm{ab}$ & $17375 \mathrm{abcd}$ & $14088 \mathrm{abcde}$ \\
$31-2-1-2$ & $36850 \mathrm{bc}$ & $28100 \mathrm{bcd}$ & $1988 \mathrm{de}$ & $5463 \mathrm{~b}$ & $15088 \mathrm{~cd}$ & $11750 \mathrm{bcde}$ \\
$4-5-1-1$ & $35975 \mathrm{bc}$ & $27425 \mathrm{~cd}$ & $3075 \mathrm{~cd}$ & $5400 \mathrm{~b}$ & $15800 \mathrm{bcd}$ & $11838 \mathrm{bcde}$ \\
$3-4-1-1$ & $35088 \mathrm{c}$ & $27700 \mathrm{~cd}$ & $2025 \mathrm{de}$ & $4888 \mathrm{~b}$ & $15675 \mathrm{bcd}$ & $12688 \mathrm{bcde}$ \\
RB-Cañero (T) & $35075 \mathrm{c}$ & $25438 \mathrm{~d}$ & $4363 \mathrm{ab}$ & $5263 \mathrm{~b}$ & $13688 \mathrm{~d}$ & $8600 \mathrm{e}$ \\
Media & 40238 & 31152 & 2758 & 6100 & 17650 & 26,4 \\
CV \% & 22,9 & 18,5 & 27,1 & & 26,2 & 28,6 \\
\hline
\end{tabular}

PTP: peso total de planta (kg/ha); PT: peso de tallo (kg/ha); PE: peso de espiga (kg/ha); PH: peso de hoja (kg/ha); PSP: peso seco de planta (kg/ha); PST: peso seco del tallo (kg/ha); CV: coeficiente de variación / PTP: total plant weight (kg/ha); PT: stem weight (kg/ ha); PE: panicle weight (kg/ha); PH: leaf weight (kg/ha); PSP: plant dry weight (kg/ha); PST: stem dry weight (kg/ha); CV: coefficient of variation.

\section{Peso de hoja (PH), peso seco de planta (PSP) y peso seco de tallo (PST)}

Potranca x Keller y la variedad 17-1-2-1 (Cuadro 3), presentaron el mayor PH con 7700 y 7400 (kg/ha), respectivamente. Asimismo, estos dos materiales presentaron valores superiores en PH, PSP y PST que RB-Cañero, y esta última variedad fue la que presentó los valores más bajos. 


\section{Peso de jugo (PJ) y ${ }^{\circ}$ Brix}

Potranca x Keller (Cuadro 4), presentó 37\% mayor producción (14 $125 \mathrm{~kg} / \mathrm{ha}$ ) de PJ que Keller (10 $325 \mathrm{~kg} / \mathrm{ha}$ ). En contenido de azúcar en el jugo en ${ }^{\circ}$ Brix $(14,8)$, fue igual que los testigos (16,4 a 16,5), excepto a RB-Cañero $\left(8,5^{\circ}\right.$ Brix $)$, el cual presentó el valor más bajo. Algunos materiales presentaron valores inferiores en PJ (7950 a 9275) y ${ }^{\circ}$ Brix $(12,4)$, como las variedades experimentales 20-3-1-1-2 y 4-5-1-1. Por otra parte, en PJ y ${ }^{\circ}$ Brix las variedades 5-5-1-1-1PL (12 438 y 14,5$), 17-1-2-1$ (11 813 y 15,5$), 22-2-2-1(11800$ y 15,5$)$ y $17-1-1-1(11750$ y 15,2$)$ fueron iguales a Potranca $x$ Keller (14 125 y 14,8), Potranca x Urja (10 163 y 14), Keller (10 325 y 16,4) y Urja (10 063 y 16,5).

Cuadro 4. Características agronómicas de diecisiete genotipos de sorgos dulces evaluados en dos localidades del noreste de México, durante los años 2013 y 2014.

Table 4. Agronomic characteristics of seventeen sweet sorghum genotypes, evaluated in two locations of northeastern Mexico, during 2013 and 2014.

\begin{tabular}{|c|c|c|c|c|c|c|c|}
\hline Genotipo & PJ & ${ }^{0}$ Brix & DF & $\mathbf{A P}$ & BI & IEF & $\mathbf{A}$ \\
\hline Potranca $x$ Keller & $14125 \mathrm{a}$ & $14,8 \mathrm{bcde}$ & $70,9 \mathrm{~g}$ & $228 \mathrm{abc}$ & $2157 \mathrm{a}$ & 2,4abcd & $2,3 \mathrm{ab}$ \\
\hline 5-5-1-1-1PL & $12438 \mathrm{ab}$ & $14,5 \mathrm{cdef}$ & $77,3 \mathrm{def}$ & $235 \mathrm{ab}$ & $1952 \mathrm{abc}$ & $2,6 a b$ & $1,8 b$ \\
\hline $17-1-2-1$ & $11813 \mathrm{abc}$ & 15,5 abcde & $81,1 \mathrm{bcd}$ & $235 \mathrm{ab}$ & 1949ab & 2,3abcd & $2,2 \mathrm{ab}$ \\
\hline $22-2-2-1$ & $11800 \mathrm{abc}$ & 15,5 abcde & 80,5 bcd & $212 \mathrm{bcd}$ & 1770abcd & $2,8 \mathrm{a}$ & $1,8 \mathrm{~b}$ \\
\hline $17-1-1-1$ & $11750 \mathrm{abc}$ & 15,2 abcde & $80,8 \mathrm{bcd}$ & 224abcd & 1929abc & $2,0 \mathrm{~d}$ & $2,2 \mathrm{ab}$ \\
\hline Keller $(\mathrm{T})$ & $10325 \mathrm{bcd}$ & $16,4 \mathrm{abcd}$ & $77,7 \mathrm{cde}$ & $224 \mathrm{abcd}$ & $1628 \mathrm{bcd}$ & $2,6 a b c$ & $3,2 \mathrm{ab}$ \\
\hline $4-2-1-1$ & $10200 \mathrm{bcd}$ & 15,9abcde & $79,5 \mathrm{bcde}$ & $225 \mathrm{abcd}$ & 1739abcd & 2,3abcd & $2,5 \mathrm{ab}$ \\
\hline Potranca x Urja & $10163 \mathrm{bcd}$ & 14,0def & $74,2 \mathrm{fg}$ & $227 \mathrm{abcd}$ & 1709abcd & 2,4abcd & $2,3 \mathrm{ab}$ \\
\hline Urja $(\mathrm{T})$ & $10063 \mathrm{bcd}$ & $16,5 \mathrm{abc}$ & $86,7 \mathrm{a}$ & $243 a$ & 1849abcd & $2,1 \mathrm{bcd}$ & $3,5 \mathrm{ab}$ \\
\hline Potranca & $9688 \mathrm{bcd}$ & $14,4 \mathrm{cdef}$ & 77,5 bcdef & $205 \mathrm{~cd}$ & $1524 \mathrm{bcd}$ & 2,5abcd & $2,3 \mathrm{ab}$ \\
\hline $20-3-1-1-2$ & $9275 b c d$ & $12,4 \mathrm{f}$ & 77,7cdef & 221abcd & $1584 \mathrm{bcd}$ & $2,7 \mathrm{ab}$ & $2,0 \mathrm{~b}$ \\
\hline Rox Orange & $8725 \mathrm{~cd}$ & $13,9 \mathrm{ef}$ & $75,6 \mathrm{~g}$ & $202 d$ & $1455 \mathrm{~cd}$ & 2,5abcd & $2,0 \mathrm{~b}$ \\
\hline $4-1-1-1$ & $8600 \mathrm{~cd}$ & $17,4 \mathrm{a}$ & $81,3 \mathrm{bc}$ & $223 \mathrm{abcd}$ & $1566 \mathrm{bcd}$ & 2,3abcd & $2,50 \mathrm{~b}$ \\
\hline $31-2-1-2$ & $7963 d$ & 15,8 abcde & $86,7 \mathrm{a}$ & $263 \mathrm{abcd}$ & $1529 \mathrm{bcd}$ & 2,3abcd & $2,0 \mathrm{~b}$ \\
\hline $4-5-1-1$ & $7950 d$ & $12,4 \mathrm{f}$ & $75,7 \mathrm{ef}$ & $229 a b c$ & $1492 \mathrm{~cd}$ & $2,0 \mathrm{~cd}$ & $2,2 \mathrm{ab}$ \\
\hline $3-4-1-1$ & $7663 d$ & $17,6 a b$ & $81,3 \mathrm{bc}$ & $223 \mathrm{abcd}$ & $1507 \mathrm{~cd}$ & 2,5abcd & $3,2 \mathrm{ab}$ \\
\hline RB-Cañero (T) & $7175 d$ & $8,5 \mathrm{~g}$ & $81,6 b$ & 222abcd & $1409 d$ & $2,0 \mathrm{~cd}$ & $3,7 \mathrm{a}$ \\
\hline Media & 9975 & 14,7 & 79,2 & 224 & 1695 & 2,37 & 2,5 \\
\hline $\mathrm{CV} \%$ & 26,08 & 12,68 & 3,70 & 8,0 & 23,9 & 15,39 & 23,67 \\
\hline
\end{tabular}

PJ: peso de jugo (kg/ha); ${ }^{\circ}$ Brix: medida del contenido de azúcar en el jugo; DF: días a flor; AP: altura de planta (cm); BI: bioethanol production (1/ha); IEF: incidencia de enfermedades foliares; A: acame, estas dos últimas características se calificaron en forma visual de mayor a menor mediante una escala de 1 al $5, \mathrm{CV}$ : coeficiente de variación / PJ: juice weight (kg/ha); ${ }^{\circ}$ Brix: measure of sugar content found in juice; DF: days to flower; AP: plant height (cm); BI: bioethanol production (1/ha); IEF: foliar diseases incidence; A: stalk lodging, the last two features were rated visually from high to low on a scale of 1 to 5, CV: coefficient of variation.

\section{Días a floración (DF) y altura de planta (AP)}

En este estudio los valores para días a floración fluctuaron entre 70,9 a 86,7 (Cuadro 4). Los materiales más precoces fueron los híbridos experimentales Potranca x Keller $(70,9)$ y Potranca x Urja $(74,2)$, y los más tardíos fueron Urja y 31-2-1-2 con 86,7 días. Potranca x Keller fue 6,8 días más precoz que Keller, 15,8 respecto a Urja y 10,7 en relación con RB-Cañero. En promedio, los genotipos presentaron alturas entre 202 a $263 \mathrm{~cm}$. 


\section{Producción de bioetanol (BI)}

Potranca x Keller (Cuadro 4) fue el genotipo que presentó el valor más alto de bioetanol (2157 1/ha), similar a las variedades experimentales 5-5-1-1-1PL, 17-1-2-1, 22-2-2-1, 17-1-1-1, 4-2-1-1 (1739 a 1952 1/ha), Urja (1849 1/ha) y Potranca x Urja (1709 1/ha), mientras que RB-Cañero presentó el valor más bajo (1409 1/ha). Potranca x Keller fue 32,5\% superior en producción de bioetanol a Keller (1628 1/ha).

\section{Incidencia de enfermedades foliares (IEF) y acame (A)}

Las variedades experimentales 17-1-1-1, 4-5-1-1 y la variedad comercial RB-Cañero (Cuadro 4), fueron más tolerantes $(2,0)$ a las enfermedades de tizón de la hoja (Helmintosporium turcicum (Pass.) Leo and Suggs), antracnosis (Colletotrichum graminicola (Ces.) Wilson), y roya (Puccinia purpurea Cooke) que Keller $(2,6)$. Para acame las variedades experimentales 5-5-1-1-1PL, 22-2-2-1, 20-3-1-1-2 y 31-2-1-2 fueron más tolerantes (1,8 a $2,0)$ que RB-Cañero $(3,7)$.

\section{Discusión}

\section{Análisis combinado}

Las diferencias altamente significativas para ambientes, genotipos y la interacción genotipo x ambiente; señalan que existió una respuesta diferencial de los genotipos en los ambientes, esto es explicable debido a las diferencias en localidad, clima, tipo de suelo, fertilización, riego, secano, fecha de siembra, etc. Las características de PJ, PTP, PT, AP, PE, PH, BI, PSP y PST (Cuadro 2), fueron superiores en ECOI-2014 y ECV-2014, esto debido a que la Estación Cuauhtémoc presenta mejores condiciones climáticas y de suelo que Marín, donde las temperaturas son más cálidas y secas, y es más común que se produzca estrés hídrico y de temperatura. En el experimento de ECV-2013, se presentaron los valores más bajos en PJ, PTP, PT, AP, PE, PH, PSP, PST y BI , debido a que después de la siembra hubo lluvias extremas, que afectaron el crecimiento de las plantas y atrasaron su desarrollo y producción. Las diferencias observadas en días a floración, entre los experimentos de la Estación Cuauhtémoc OI-2014 $(83,4)$ y V-2013 $(84,8)$ (Cuadro 2), se debieron a que, en el primer caso, el cultivo se desarrolló durante el invierno, donde estuvieron presentes temperaturas más bajas (media $21,5^{\circ} \mathrm{C}$ ). La segunda fue porque las plantas retrasaron su ciclo, debido a las abundantes lluvias que ocurrieron después de la siembra (390 mm). En el caso de las diferencias observadas en días a floración entre MP-2014 (82,4) y MV-2014 $(69,7)$, se debieron a que, en este último experimento, el cultivo prosperó durante un ciclo y ambiente más cálido (media $27,0{ }^{\circ} \mathrm{C}$ contra media del $18,5^{\circ} \mathrm{C}$ ). Al respecto, House (1985) indicó que el sorgo S. bicolor no es un cultivo tolerante al frío, por lo que, crece lentamente en temperaturas de $20^{\circ} \mathrm{C}$.

El ambiente juega un papel importante en la producción de azúcar. Reportes indican que el sorgo dulce es muy eficiente en ambientes cálidos (Rooney y Serna, 2000; Almodares y Hadi, 2009) y con intensidad luminosa alta (Rooney y Serna 2000). También en ambientes más secos, es mayor la producción de azúcar (Almodares y Hadi, 2009). Se encontró un $29 \%$ mayor producción de azúcar en los sorgos evaluados en condiciones de secano que en riego (Alhajturki et al., 2012). La localidad de Marín tuvo un clima cálido-seco y la Estación Cuauhtémoc un clima cálido subhúmedo, lo que explica los valores más altos obtenidos en ${ }^{\circ}$ Brix en MP-2014 (16,2), y los más bajos encontrados en ECOI-2014 (14,3) y ECV-2013 $(14,6)$. 


\section{Peso total de planta (PTP), peso de tallo (PT) y Peso de espiga (PE)}

La explicación de la superioridad de Potranca x Keller en PTP, PT y PE (Cuadro 3), es el grado de heterosis que presentó esta combinación híbrida. La heterosis se define como la superioridad del híbrido en porcentaje sobre la media de los padres (León-Velasco et al., 2009). En el híbrido experimental Potranca x Keller; Potranca fungió como progenitor femenino y Keller como masculino. El híbrido presentó una heterosis de 28,6, 19,4 y $42 \%$ respectivamente para PTP, PT y PE. Esto concuerda con lo reportado en sorgo, donde se indica que, en general, los híbridos son mejores que sus progenitores (Williams y Arcos, 2015). Pfeiffer et al. (2010) indicaron que, aunque la heterosis está bien confirmada en sorgos de grano (S. bicolor) y forrajero (Sorghum sp.), los reportes en sorgo dulce se han limitado a cruzas entre sorgo de grano x sorgo dulce. Potranca x Keller es un híbrido en el que ambos progenitores son dulces, y confirman las tendencias encontradas en las cruzas de sorgos de grano x dulces. Sorgos dulces con alta producción de biomasa son los preferidos (Pothisoong y Jaisil, 2011; Elangovan et al., 2014; Mishra, et al., 2015).

Los sorgos dulces pueden producir entre 58300 a $80500 \mathrm{~kg} / \mathrm{ha}$ de biomasa (Sylvester et al., 2015), en tanto que Pothisoong y Jaisil (2011) reportaron producciones entre 48300 y $50800 \mathrm{~kg} / \mathrm{ha}$. Potranca x Keller produjo en promedio $52125 \mathrm{~kg} / \mathrm{ha}$ de PTP, resultado que se encuentra entre el nivel inferior reportado por los primeros autores y el superior indicado por los segundos. Las diferencias encontradas se pueden deber a condiciones agroclimáticas diferentes: sequía, localidad, suelos, clima, manejo agronómico y genotipos. Los menores valores de Rox Orange (Cuadro 4) se pueden deber a que fue el genotipo que resultó con menor altura de planta. Se ha encontrado correlación positiva entre altura de planta y producción de biomasa (Murray, 2008); o sea que, cuando se presenta menor altura de planta, menor será la producción de biomasa. El mayor peso de espiga de Potranca x Keller, se debe al efecto del vigor híbrido, estimándose una heterosis de 99,4\%. Al respecto, diversos autores han encontrado heterosis para rendimiento de grano (Cisneros et al., 2007; Pothisoong y Jaisil, 2011; Williams y Arcos, 2015), característica que es muy similar al dato de PE tomado en este estudio.

\section{Peso de hoja (PH), peso seco de planta (PSP) y peso seco de tallo (PST)}

RB-Cañero fue la variedad que presentó los valores más bajos para peso de hoja, peso seco de planta y peso seco de tallo, debido a que fue la variedad que presentó los valores más bajos en peso seco total de planta y peso de tallo.

\section{Peso de jugo (PJ) y ${ }^{\circ}$ Brix}

La mayor producción de jugo de Potranca x Keller (14 $125 \mathrm{~kg} / \mathrm{ha}$ ) se asoció a una heterosis de 29,2\% (Cuadro 4). De acuerdo con Murray (2008), el peso de jugo y ${ }^{\circ}$ Brix son características de suma importancia, porque son determinantes en la producción de bioetanol por hectárea. La selección de genotipos para alta producción de jugo, ${ }^{\circ}$ Brix y total de azúcares solubles es esencial (Elangovan et al., 2014). Los sorgos dulces presentan valores de azúcar en el jugo entre 12,4 a $24^{\circ}$ Brix (Elangovan et al., 2014), con lo cual los sorgos evaluados en este estudio, corresponden a esta clasificación, excepto RB-Cañero. Debido a los resultados obtenidos con el híbrido experimental Potranca x Keller en: PTP, PT, PH, PSP, PST, PJ y ${ }^{\circ}$ Brix, se encuentra en proceso de registro en el catálogo de variedades de plantas con el nombre de Potrillo.

\section{Días a floración (DF) y altura de planta (AP)}

Los valores para la floración en $S$. bicolor pueden variar entre 52,4 a 90,5 días. En este estudio los valores para días a floración se encontraron dentro de los rangos antes descritos (Cuadro 4). Los resultados están de acuerdo con 
los reportes de que, en general, los híbridos presentan heterosis negativa y son más precoces que el promedio de sus padres (Mahdy et al., 2011; Williams y Arcos 2015). Potranca x Keller presentó una heterosis para días a floración de $-9,4 \%$, información similar a la encontrada por Pothisoong y Jaisil (2011), quienes reportaron una heterosis de $-7,83 \%$. Las variedades tardías Urja y 31-2-1-2 no reflejaron una mayor producción de jugo y biomasa. Los resultados contrastan con los de Zhang y Wang (2015), quienes indicaron que en el sorgo dulce, los genotipos tardíos generalmente presentan mayor producción de biomasa. Por otra parte, se reportó que los sorgos S. bicolor de ciclo precoz, presentan ventajas en condiciones de sequía y altas temperaturas cuando la estación de crecimiento es corta (Murphy et al., 2014). También pueden ser útiles para reducir el intervalo de tiempo entre un cultivo y el siguiente. Las condiciones climáticas que se presentan en la región de las Huastecas son irregulares, por lo que, con frecuencia se presenta condiciones de sequía y altas temperaturas (Williams y Arcos, 2015), por lo cual, la precocidad del híbrido Potranca x Keller es una característica importante para su cultivo y producción en condiciones de escasa humedad.

Altura de planta es un importante componente agronómico que incide en la producción de biomasa. En los sorgos dulces las plantas más altas parecen ser la llave para obtener una mayor producción de biomasa y azúcar. Los sorgos dulces S. bicolor, frecuentemente presentan alturas de planta superiores a $3 \mathrm{~m}$ (Sylvester et al., 2015) (Cuadro 4). Esto depende de las condiciones de clima, suelo, manejo del cultivo, genotipo, etc. Los genotipos con menor altura de planta fueron las variedades Rox Orange $(202 \mathrm{~cm})$ y Potranca $(205 \mathrm{~cm})$. También se relaciona a que fueron los más precoces y los que presentaron la menor producción de PTP, PT, PE y PJ.

\section{Producción de bioetanol (BI)}

En este trabajo se encontró una producción promedio de etanol de 1695 1/ha, con un rango entre 1409 a 2157 1/ha (Cuadro 4). Reportes indican que el sorgo dulce cultivado bajo buenas condiciones agroclimatológicas, puede producir hasta 98 t/ha de tallo fresco, suficiente para obtener entre 3700 a 48001 de etanol por hectárea (Hills et al., 1990). En Damasco, Siria se evaluaron nueve genotipos dulces bajo dos condiciones de humedad, con un potencial para producir etanol entre 264 a 3054 1/ha (Alhajturki et al., 2012). En Irán se reporta una producción promedio de sorgo dulce en riego en cuatro meses, entre 54 a 69 t/ha de peso de biomasa y una producción promedio de etanol de 3000 1/ha (Almodares y Hadi, 2009). Se considera que los valores obtenidos en este estudio se encuentran dentro de los resultados esperados en localidades de temporal o en ambientes con suelos pobres. En este caso los tres experimentos sembrados en la Estación Cuauhtémoc fueron de temporal, y los dos experimentos en Marín fueron sembrados en suelos delgados, con bajos contenidos de materia orgánica.

La explicación del valor más alto obtenido por el híbrido Potranca x Keller, se encuentra en la heterosis de 19,5\% que presentó esta combinación híbrida. De acuerdo con los resultados encontrados en este estudio, Potranca x Keller constituye la mejor opción para producir etanol, ya que, fue superior a los testigos Keller y RB-Cañero e igual que Urja, además, fue más precoz a la floración.

\section{Incidencia de enfermedades foliares (IEF) y acame (A)}

Hubo incidencia de presencia de tizón de la hoja (H.turcicum), antracnosis (C. graminicola), mancha zonada (Gleocercospora sorghi D. Bain \& Edg), roya (P. purpurea) y mancha parda (Cercospora sorghi Elis \& Everhart). Estos patógenos en el cultivo comercial de S. bicolor, son un problema en el sur de Tamaulipas (Williams y Arcos, 2015). Se reporta que el ataque de mancha ovalada de la hoja (Ramulispora sorghicola Harris) y mancha gris $(C$. sorghi), pueden resultar en defoliación prematura de la planta, reducción del contenido de azúcar en el tallo y la disminución del rendimiento de grano (Girad, 1980). La defoliación debido a la antracnosis (C. graminicola), reduce el valor de las plantas para el forraje, puede reducir el contenido de azúcar de los tallos de las variedades muy susceptibles y disminuir la proporción de sacarosa para invertir azúcares (Khawasja et al., 2014). 
Se indica que el mejoramiento de S. bicolor para resistencia a enfermedades, es importante para lograr un mayor aumento (Reddy et al., 2006) y estabilidad en la producción (Rooney, 2004), además de que la resistencia genética de la planta hospedante es el método más económico y exitoso en el combate de estos patógenos (Khawaja et al., 2014). En las áreas donde se pretende sembrar S. bicolor para producir etanol en México, que son Veracruz, Jalisco, Chiapas, Tabasco, Tamaulipas y Colima (Zafranet, 2015), presentan un ambiente cálido y húmedo, adecuado para que se presenten las enfermedades foliares. En estos ambientes se han identificado la presencia de tizón de la hoja (H. turcicum), mancha parda (C. sorghi), mancha zonada (G. sorghi), antracnosis (G. sorghi), rayado foliar (Pseudomonas andropogonis (Smith) Stapp), roya (P. purpurea) y mildiu velloso (Peronosclerospora sorghi (Weston y Upal) C.G. Shaw) (Stack, 2002). Esto resalta la relevancia de realizar mejoramiento genético en sorgo dulce, contra este tipo de patógenos. Por su tolerancia a las enfermedades foliares y sus características agronómicas competitivas, la variedad experimental 17-1-1-1 está en proceso de registro con el nombre de Dulcina. La tolerancia al acame es una característica muy importante (Mishra et al., 2015), ya que el vuelco de las plantas puede reducir parcial o totalmente la producción de biomasa.

\section{Conclusiones}

Hubo diferencias altamente significativas para ambientes, genotipos y la interacción ambientes x genotipos, para las características de peso fresco total de planta, peso fresco de tallo, peso de espiga, peso fresco de hoja, peso seco de planta, peso seco de tallo, peso de jugo, ${ }^{\circ}$ Brix, producción de bioetanol, altura de planta, días a floración e incidencia de enfermedades foliares.

Potranca x Keller fue el genotipo que presentó los valores más altos en las características agronómicas asociadas a la producción de bioetanol: peso total de planta (52 $125 \mathrm{~kg} / \mathrm{ha})$; de tallo (35 438 kg/ha), de hoja (7700 $\mathrm{kg} / \mathrm{ha}$ ) y de jugo (14125 kg/ha); además fue más precoz a la floración. Se estima que el híbrido produjo 2157 1/ha de etanol, superior a los testigos Keller (1628 1/ha) y RB-Cañero (1409 1/ha) y fue similar a Urja con 1849 1/ha.

\section{Literatura citada}

Alhajturki D., M. Aljamali, A. Kanbar, and F. Azmah. 2012. Potential of some sweet sorghum (Sorghum bicolor L.) genotypes under two water regimes for sugar and bio-ethanol production. Sugar Tech. 14:376-382. doi:10.1007/S12355-012-0181-x

Ali, M.L., J.F. Rajewski, P.S. Baenziger K.S. Gill, K.M. Eskridge, and I. Dweikat. 2008. Assessment of genetic diversity and relationship among a collection of US sweet sorghum germplasm by SSR markers. Mol. Breeding 21:497-509. doi:10.1007/s11032-007-9149-z

Almodares, A., and M.R. Hadi. 2009. Production of bioethanol from sweet sorghum. Afr. J. Agric. Res. 4:772-780.

Almodares, A, M.R. Hadi, and B. Dosti. 2007. Effects of salt stress on germination percentage and seedling growth in sweet sorghum cultivars. J. Biol. Sci. 7:1492-1495. doi:10.3923/jbs.2007.1492.1495

Chuck, C., E. Pérez, E. Heredia, y S.O. Serna. 2011. Sorgo como un cultivo multifacético para la producción de bioetanol en México: Tecnologías, avances y áreas de oportunidad. Rev. Mex. Ing. Quim. 10:529-549.

Cisneros, M.E., L.E. Mendoza, G. Mora, L. Córdoba, y M. Livera. 2007. Híbridos y progenitores de sorgo tolerantes al frío. II: Efecto de Fusarium verticilloides (Sacc) Nirenberg en el rendimiento de semilla y sus componentes en condiciones de campo. Agrociencia 41:283-294. 
Dávila, F.J., C. Chuck, E. Pérez, W.L. Rooney, and S.O. Serna. 2011. Evaluation of bioethanol production from five different varieties of sweet and forage sorghums (Sorghum bicolor (L) Moench). Ind. Crops Prod. 33:611-616. doi:10.1016/j. indcrop.2010.12.022

Elangovan, M., P. Kiran-Babu, N. Seetharama, and J.V. Patil. 2014. Genetic diversity and heritability characters associated in sweet sorghum [Sorghum bicolor (L.) Moench]. Sugar Tech. 16:200-210. doi:10.1007/s12355-013-0262-5

García, E. 1988. Modificaciones al sistema de clasificación climática de Köppen. Universidad Nacional Autónoma de México, México, D.F., MEX.

Girard, J.C. 1980. A review of souty stripe and rouge, zonate and oval leaf spots. In: R.J. Williams et al., editors, Sorghum diseases, a world review. ICRISAT, Patancheru (AP), IND. p. 229-239.

Hills, F.J., R.T. Lewellen, and I.O. Skoyen. 1990. Sweet sorghum cultivars for alcohol production. California Agric. 44:14-16.

House, L.R. 1985. A guide to sorghum breeding. ICRISAT, Andhra Pradesh, IND.

INIFAP (Instituto Nacional de Investigaciones Forestales, Agrícolas y Pecuarias). 2013. Campo Experimental Huastecas. http:// www.inifapcirne.gob.mx/LocalizacionHuastecas.htm (consultado 9 mar. 2016).

Khawaja, C., R. Janssen, D. Rutz, D. Luquet, G. Trouche, B. Reddy, P.S. Rao, G. Basavaraj, R. Schaffert, C. Damasceno, R. Parella, A. Zacharias, R. Bushmann, N. Rettenmaier, G. Reinhardt, A. Monti, W.Z. Lizarazu, S. Amaducci, A. Marocco, W. Snijman, N. Shargie, H. Terblanche, F. Zavala-García, y S. Braconnier. 2014. Manual del sorgo dulce: Cultivo con potencial energético. http://www.sweetfuel-project.eu/publications/energy_sorghum_handbook_spanish_version (consultado 20 jun. 2016).

Mahdy, E.E., M.A. Ali, and A.M. Mahmoud. 2011. The effect of environment on combining ability and heterosis in grain sorghum (Sorghum bicolor L. Moench). Asian J. Crop Sci. 3:1-15. doi:10.3923/ajcs.2011.1.15

Mishra, J.S., N.S. Thakur, Kewalanand, P. Sujathamma, B.B. Kushwaha, S.S. Rao, and J.V. Patil. 2015. Response of sweet sorghum genotypes for biomass, grain yield and ethanol production under different fertility levels in rainfed conditions. Sugar Tech. 17:204-209. doi:10.1007/s12355-014-0315-4

Montes, N., V. Pecina, M.E. Cisneros, y M.A. García. 2010. RB Cañero: sorgo dulce [Sorghum bicolor (L.) Moench] para la producción de etanol. MEX. http://biblioteca.inifap.gob.mx:8080/xmlui/bitstream/handle/123456789/2715/867. pdf?sequence $=1$ (consultado 23 mar. 2017).

Montes, N., H. Williams, G. Arcos, V. Pecina, M. De-la-Garza, T. Moreno, y E. Vargas. 2014. RB-Huasteco, sorgo de grano para áreas de riego y buen temporal. Rev. Mex. Cienc. Agríc. 5:1331-1335.

Murphy, R.L., D.T. Morishige, J.A. Brady, W.L. Rooney, S. Yang, P.E. Klein, and J.E. Mullet. 2014. Ghd7 (Ma6) represses sorghum flowering in long days: Ghd7 alleles enhance biomass accumulation and grain production. The Plant Genome 7(2). doi:10.3835/plantgenome2013.11.0040

Murray, S.C. 2008. Genetic and phenotypic diversity in sorghum for improvement as a biofuel feedstock. PhD. Tesis, Cornell University, Ithaca, NY, USA.

Murray, SC., W.L. Rooney, MT. Hamblin, S.E. Mitchell, and S. Kresovich. 2009. Sweet sorghum genetic diversity and association mapping for brix and height. The Plant Genome 2:48-62. doi:10.3835/plantgenome2008.10.0011

León-Velasco, H., L.E. Mendoza-Onofre, F. Castillo-González, T. Cervantes-Santana, y A. Martínez-Garza. 2009. Evaluación de dos generaciones de híbridos y progenitores de sorgo tolerantes al frío. II: Aptitud combinatoria, heterosis y heterobeltiosis. Agrociencia 43:609-623.

PEMEX. 2015. Suscribe Pemex contrato para la adquisicion de etanol anhidro. www.pemex.com/saladeprensa/boletines_ nacionales/.../2015-036-nacional.aspx (consultado 12 mar. 2017). 
Pfeiffer, T.W., M.J. Bitzer, J.J. Toy, and J.F. Pedersen. 2010. Heterosis in sweet sorghum and selection of a new sweet sorghum hybrid for use in syrup production in Appalachia. Crop Sci. 50:1788-1794. doi:10.2135/cropsci2009.09.0475

Pothisoong, T., and P. Jaisil. 2011. Yield potential, heterosis and ethanol production in F1 hybrids of sweet sorghum (Sorghum bicolor L. Moench). KMITL Sci. Tech. J. 11.17-21. (accessed 1 feb. 2016).

Ratnavathi, C.V., K. Suresh, B.S. Vijay-Kumar, M. Pallavi, V.V. Komala, and N. Seetharama. 2010. Study on genotypic variation for ethanol production from sweet sorghum juice. Biomass Bioen. 34:947-952. doi:10.1016/j.biombioe.2010.02.002

Reddy, B.V.S., S. Ramesh, P.S. Reddy, B. Ramaiah, P.M. Salimath, and R. Kachapur. 2005. Sweet sorghum a potential alternate raw material for bio-ethanol and bio-energy. ISMN 46:79-86.

Reddy, B.V.S., A.A. Kumar, and W.D. Dar. 2006. Overview of sweet sorghum breeding at ICRISAT: Opportunities and constraints. https://www.ifad.org/documents/10180/eedbb75a-9f8f-4f5a-9d18-4a42cbbe1dd7 (accessed 20 Dic. 2015).

Rooney, W.L., and S.O. Serna. 2000. Sorghum. In: K. Kulp, and J. Ponte, editors, Handbook of cereal science and technology, Marcel Dekker, NY, USA. p. 149-176.

Rooney, W.L. 2004. Sorghum improvement-integrating traditional and new technology to produce improved genotypes. Adv. Agron. 83:37-109. doi:10.1016/S0065-2113(04)83002-5

Reyes, C.P. 1985. Diseño de experimentos aplicados. $4^{a}$ ed. Ed. Trillas, México D.F., MEX.

Serna, S.O. 2010. Cereal grains: properties, processing, and nutritional attributes. CRC Press, Boca Raton, FL, EUA.

SIAP (Servicio de Información Agroalimentaria y Pesquera). 2017. Cierre de la produccion agrícola por cultivo (serie 1980 a $2015)$ http://infosiap.siap.gob.mx/aagricola_siap_gb/ientidad/index.jsp (consultado 5 ago. 2017).

Stack, J.P. 2002. Recurring and emerging sorghum diseases in North America. In: J.F. Leslie, editor, Sorghum and millets disease. Iowa State Press, Ames, IA, USA. p. 449-456.

Stephens, J.C., and R.F. Holland. 1954. Cytoplasmic male sterility for hybrids sorghum seed production. Agron. J. 46:20-23.

Sun, Y., G. Niu, P. Osuna, L. Zhao, G. Ganjegunte, G. Peterson, J.R. Peralta-Videa, and J.L. Gardea-Torresdey. 2014. Variability in salt tolerance of Sorghum bicolor L. Agric. Sci. 2:9-21.

Sylvester, E.A., Z. Li-Min, X. Yan, Z. Yu-Miao, L. Zhi-Quan, and J. Hai-Chun. 2015. Sweet sorghum ideotypes: genetic improvement of the biofuel syndrome. Food Energy Security. Food an Energy Security 4:3-24. doi:10.1002/fes3.63

Tobin. 2010. El sorgo azucarado un cultivo energético promisorio para el NOA. http://www.tobin.com.ar/?El_Sorgo_ Azucarado\%3A_Un_cultivo_energ\%E9tico_promisorio_para_el_NOA\&page=ampliada\&id=54\&_s=\&_page= (consultado 23 mar. 2016).

Undersander, D.J., W.E. Lueschen, L.H. Smith, A.R. Kaminski, J.D. Doll, K.A. Kelling, and E.S. Oplinger. 1990. Sorghum syrup. corn.agronomy.wisc.edu/Crops/SorghumSyrup.aspx (accessed 20 apr. 2016).

Vanderlip, R.L. 1993. How a sorghum plant develop. Contribution No. 1203. Kansas Agricultural Experiment Station, Manhattan, KS, USA.

Vietor, D.M., and F.R. Miller. 1990. Assimilation, partitioning, and nonstructural carbohydrate in sweet compared with grain sorghum. Crop Sci. 30:1109-1115.

Williams, H., y G. Arcos. 2015. Comportamiento agronómico de híbridos y progenitores de sorgo para grano en las Huastecas. Agron. Mesoam. 26:87-97. doi:10.15517/am.v26i1.16926

Williams, H., N. Montes, y V. Pecina. 2006. Sorgo. En: L.A. Rodríguez-del-Bosque, editor, 50 años de investigación agropecuaria en el norte de Tamaulipas, historia, logros y retos. Libro técnico No. 1. INIFAP, Río Bravo, Tamaulipas, MEX. p. 33-54. 
Williams, H., V. Pecina, F. Zavala, y N. Montes. 2004. RB-Patrón, nuevo híbrido de sorgo para grano en el noreste de México. Rev. Fitotec. Mex. 27:291-293.

Yuvraj, G., R. Kaur, S.K. Uppal, P. Sharma, and H.S. Oberoi. 2013. Chemical composition of sweet sorghum juce and its comparative potential of different fermentation processes of enhanced etanol production. Sugar Tech. 15:305-310. doi:10.1007/S12355-013-0232-y

Zafranet. 2015. Impulsan la producción de biocombustibles a partir de caña, sorgo, agave y remolacha. Zafranet, MEX. www. zafranet.com/2015/12/etanol-cana-sorgo-agave-remolacha (consultado 18 feb. 2016).

Zhang, B., and Q. Wang. 2015. MicroRNA-based biotechnology for plant improvement. J. Cell. Physiol. 230:1-15. doi:10.1002/ jсp. 24685 